\title{
Structure change, layer sliding, and metallization in high-pressure $\mathrm{MoS}_{2}$
}

\author{
Liliana Hromadová, ${ }^{1,2}$ Roman Martoňák, ${ }^{1, \text { f }}$ and Erio Tosatti²,3 \\ ${ }^{1}$ Department of Experimental Physics, Comenius University, \\ Mlynská Dolina F2, 84248 Bratislava, Slovakia \\ ${ }^{2}$ International School for Advanced Studies (SISSA) and CNR-IOM Democritos, \\ Via Bonomea 265, I-34136 Trieste, Italy \\ ${ }^{3}$ The Abdus Salam International Centre for Theoretical Physics (ICTP), \\ Strada Costiera 11, I-34151 Trieste, Italy
}

(Dated: October 30, 2018)

\begin{abstract}
Based on ab initio calculations and metadynamics simulations, we predict that $2 \mathrm{H}-\mathrm{MoS}_{2}$, a layered insulator, will metallize under pressures in excess of 20-30 GPa. In the same pressure range, simulations and enthalpy optimization predict a structural transition. Free mutual sliding of layers takes place at this transition, the original $2 \mathrm{H}_{c}$ stacking changing to a $2 \mathrm{H}_{a}$ stacking typical of $2 \mathrm{H}-\mathrm{NbSe}_{2}$, an event explaining for the first time previously mysterious X-ray diffraction and Raman spectroscopy data. Phonon and electron phonon calculations suggest that pristine $\mathrm{MoS}_{2}$, once metallized, will require ultrahigh pressures in order to develop superconductivity.
\end{abstract}

PACS numbers: $61.50 . \mathrm{Ks}, 71.30 .+\mathrm{h}, 71.15 . \mathrm{Pd}, 74.62 . \mathrm{Fj}$ 
Transition metal dichalcogenides $\mathrm{MX}_{2}$, where $\mathrm{M}$ is a transition metal and $\mathrm{X}=\mathrm{S}$, Se and Te, are layered materials displaying a variety of electronic behavior from insulating to chargedensity-wave to metallic to superconducting, with a rich scenario of phase transitions as a function of external parameters. Intercalation of electron-donating atomic species between the weakly bonded $\mathrm{MX}_{2}$ layers is facile, turning the insulators to metals which are interesting catalysts, and which also often superconduct at cryogenic temperatures. Perhaps the best known case in point is that of $\mathrm{MoS}_{2}$, a semiconductor with an indirect electronic band gap of $1.29 \mathrm{eV}$ [1], which in its pristine state is well known as a lubricant [2], and more recently as a potential photovoltaic [3] and single-layer transistor [4] material. $\mathrm{MoS}_{2}$ metallizes under intercalation of alkalis and alkaline earths [5], achieving superconducting temperatures up to $6 \mathrm{~K}$. It has very recently also been shown to superconduct at even higher temperatures upon electric double layer (EDL) field doping [6, 7].

High pressure provides, besides doping, another general route to transform insulators to metals. Recently it was shown that bilayer sheet of $\mathrm{MoS}_{2}$ undergoes semiconductor-metal transition upon vertical compressive pressure [8]. The possibility that $\mathrm{MoS}_{2}$ might metallize under pressure is also suggested by a negative pressure coefficient of resistivity [9] indicating gap shrinking, $d E_{G} / d P<0$. Whether and how high pressure gap closing and metallization in bulk could be achieved is, however, an open question, also because X-ray diffraction [10] and Raman spectroscopy [11] data indicate a structural transition taking place between 20 and $30 \mathrm{GPa}$ to another phase of unknown structure and properties. What is the structural transition, whether high pressure metallization and possibly superconductivity will eventually occur or not, and what could be the interplay among these structural and electronic phenomena in an initially insulating material, are all open questions of considerable interest, for which $\mathrm{MoS}_{2}$ can play a prototypical role. We alternated and combined well established first principles density functional theory (DFT) calculations and ab initio metadynamics (AIMtD) simulations [12, 13] to predict and clarify the simultaneous evolution of structural and electronic properties of pristine $\mathrm{MoS}_{2}$ under pressure.

AIMtD simulation - where the supercell parameters act as collective variables - is a powerful computational tool to discover new and competing solid phases [14, 15]. For ab initio molecular dynamics and structural relaxations we employed VASP (Vienna ab initio simulation package) [16] with standard scalar relativistic PAW pseudopotentials (no spinorbit included)[17] and cutoff of $340 \mathrm{eV}$. MD simulations were performed using a 72-atoms 
$2 \sqrt{(3)} \times 2 \sqrt{(3)}$ supercell and $2 \times 2 \times 2$ Monkhorst-Pack[18] (MP) k-point sampling grid. For structural relaxations we used the 6 -atoms unit cell and a $9 \times 9 \times 5$ MP grid. The electronic structure calculations and structural optimizations were independently conducted using a variety of exchange-correlation functionals: LDA, GGA with PBE parametrization [19], hybrid functionals B3LYP [20, 21] and HSE06 [22]. Van der Waals interactions were included in the Grimme approximation [23] in the initial calculations at zero pressure, where they made an important contribution to the interplanar attraction, but were subsequently removed at high pressures. It turned out that at pressures above $5 \mathrm{GPa}$, in a regime where interlayer interactions are repulsive it is GGA with PBE potential and without van der Waals corrections that provides lattice parameters closest to experimental data.

Within that approximation we thus carried out accurate total energy-based structural relaxations at increasing pressures, refining the properties of the phase(s) discovered with AIMtD dynamical simulations. That combined approach produced the results which we now describe.

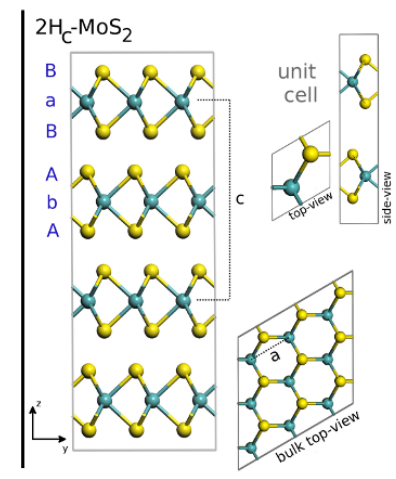

(a)

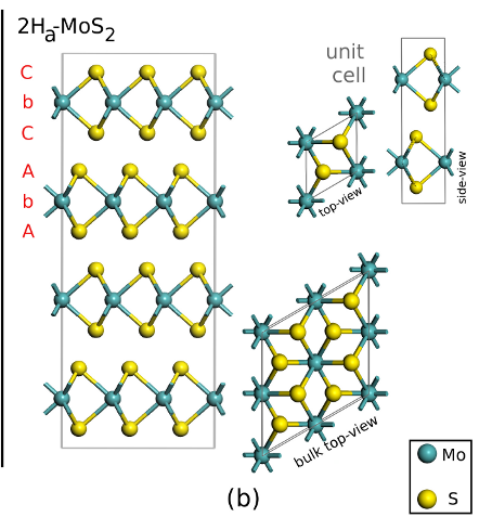

(b)

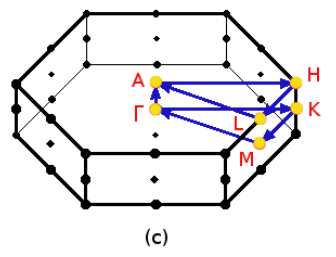

FIG. 1: Structures of $2 \mathrm{H}_{c}-\mathrm{MoS}_{2}$ (a) and $2 \mathrm{H}_{a}-\mathrm{MoS}_{2}$ (b). I. Brillouin zone for both structures 24] (c).

Static relaxation of the initial structure $2 \mathrm{H}_{c}-\mathrm{MoS}_{2}$ (space group $P 6_{3} / m m c$ ) of Fig.1 (a) [32] upon increasing pressure produced no instability towards other phases up to $50 \mathrm{GPa}$. The unit cell dimensions shrank anisotropically (Fig 2) as expected from a softer c-axis and 
harder a,b axes. The indirect electronic band gap also shrank as expected, and eventually closed near $25 \mathrm{GPa}$ with an electron-hole wavevector $\delta k \approx \frac{1}{2} K$ (Fig, 3 ) where $K=\left(\frac{1}{3}, \frac{1}{3}, 0\right)$ is a Brillouin Zone edge point (Fig.1(c)) thus predicting band overlap metallization of $2 \mathrm{H}_{c^{-}}$ $\mathrm{MoS}_{2}$ above $25 \mathrm{GPa}$. This result agrees with the very recent finding in Ref.[25]. Alternative DFT calculations and relaxations carried out with the HSE06 [22] and the B3LYP [20, 21] approximations yielded a metallization pressure of 35 and $55 \mathrm{GPa}$, respectively. Since these approaches, especially the latter, are known to underestimate metallicity, whereas GGA/PBE may overestimate it, we conclude that pressure induced metallization of $2 \mathrm{H}_{c^{-}}$ $\mathrm{MoS}_{2}$ should be placed between 25 and 35 GPa.

Extending in this pressure region our calculations to the 72 -atoms $2 \sqrt{(3)} \times 2 \sqrt{(3)}$ supercell in place of the initial 6-atom unit cell, we further explored the possible onset of a periodic lattice distortion or charge-density-wave phase (more properly called an "excitonic insulator" [26]). We performed structural optimizations of samples with small random displacements of atoms and looked for the possible onset of the accompanying periodic lattice distortion, but failed to find any. In fact, the possible occurrence of an excitonic insulator driven superstructure in a narrow pressure interval near the insulator-metal transition, an occurrence which can be considered quite probable, cannot seriously be established within our methods, and more refined treatments of exchange will be called for in the future.

We focused so far on the pressure evolution of the $2 \mathrm{H}_{c}-\mathrm{MoS}_{2}$ initial structure. However, the local stability which we found upon relaxation is no guarantee for global stability at higher pressures. The AIMtD simulations, where the supercell vectors are biased away from the initial structure towards newer ones, can reveal the possible structures missed by straight relaxation. When applied to $2 \mathrm{H}_{c}-\mathrm{MoS}_{2}$ in the 72 -atom supercell at $p=40 \mathrm{GPa}$, it led to two successive and surprising sliding events shown in Fig.4. In the first event after metastep 73 the two layers within the supercell shifted with respect to each other so that the Mo atom $(x, y)$ coordinates in the two layers became coincident (Fig,4(a)). At the same time the supercell developed a tilt: the $\alpha$ and $\beta$ angles moved away from right angle as can be seen in Fig.4(c). In this intermediate structure the parameter $a$ increased and the parameter $c$ decreased from their original values (Fig $4(\mathrm{~b})$ ). In the second event following metastep 125 the $\alpha$ and $\beta$ angles returned to the original 90 degrees while the parameters $a$ and $c$ further increased and decreased, respectively. The final structure was relaxed at $p=40$ GPa and $\mathrm{T}=0$ and was identified as $2 \mathrm{H}_{a}$ - another $2 \mathrm{H}$ polytype with the same space group, 

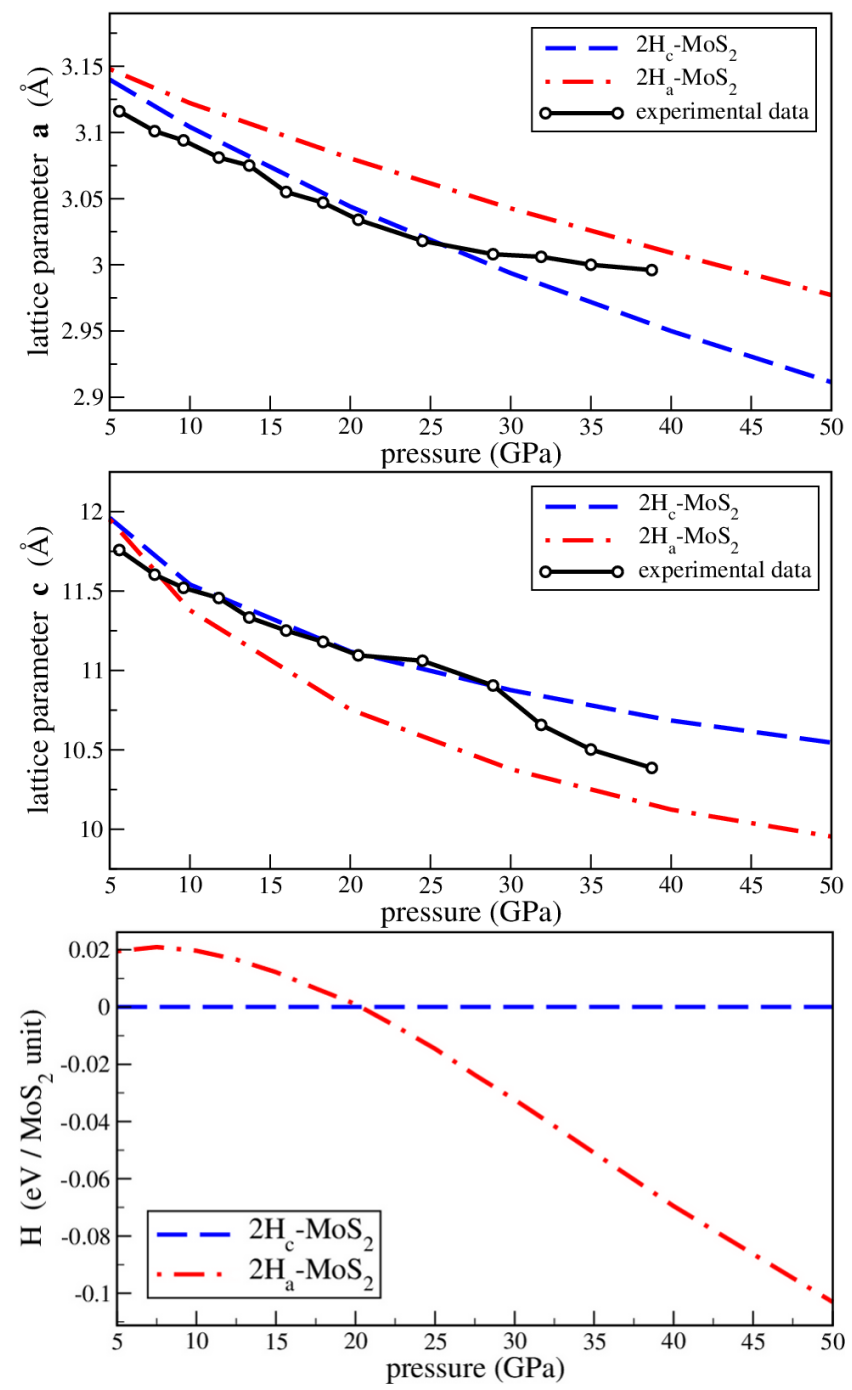

FIG. 2: Calculated lattice parameters $a$ (up) and $c$ (middle), and relative enthalpies (bottom) of $2 \mathrm{H}_{a^{-}}$and $2 \mathrm{H}_{c^{-}}-\mathrm{MoS}_{2}$ structures as a function of pressure. The experimental data taken from Table 1 in Ref.[10] are in good agreement with calculated crossing of enthalpies at $p \sim 20 \mathrm{GPa}$ as shown in the bottom picture.

but where the layer stacking is now $\mathrm{AbA} \mathrm{CbC}$ in place of the initial $\mathrm{AbA} \mathrm{BaB}$ stacking of $2 \mathrm{H}_{c}[27,28]$. In the new structure - typical of e.g., $2 \mathrm{H}-\mathrm{NbSe}_{2}-$ all Mo atoms share the same $(x, y)$ coordinates. Thus the pressure induced easy sliding of layers - a "superlubric" event, possibly related to $\mathrm{MoS}_{2}$ 's lubricant properties - transformed the $2 \mathrm{H}_{c}$ structure of $\mathrm{MoS}_{2}$ to $2 \mathrm{H}_{a}$.

Through accurate structural relaxations and electronic structure calculations we refined the cell parameters, the enthalpy and the electronic band structure of $2 \mathrm{H}_{a}-\mathrm{MoS}_{2}$, with results 

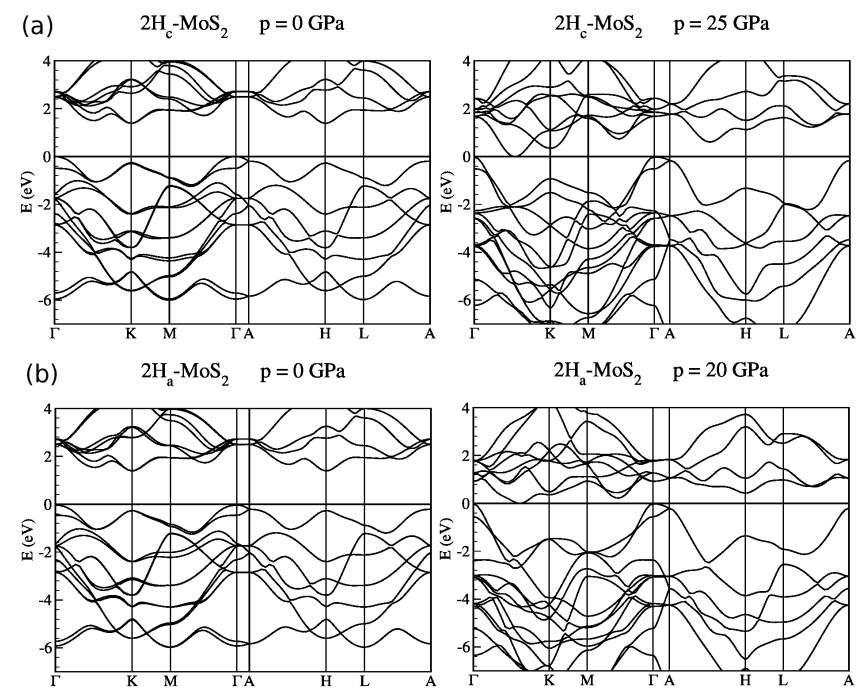

FIG. 3: Calculated band structures of $2 \mathrm{H}_{c^{-}}$(a) and $2 \mathrm{H}_{a^{-}}-\mathrm{MoS}_{2}$ (b) at selected pressures.

shown in Figs. 2, 3. The $2 \mathrm{H}_{c}$ and $2 \mathrm{H}_{a}$ enthalpies cross near $20 \mathrm{GPa}$, justifying the structural transition discovered by AIMtD. 33. The new $2 \mathrm{H}_{a}-\mathrm{MoS}_{2}$ structure has at $p=20$ GPa lattice parameters $a=3.080 \AA$ A,$c=10.754 \AA$ with Mo atoms at Wyckoff position $2(\mathrm{~b})$ and $\mathrm{S}$ atoms at $4(\mathrm{f})$ with $z=0.8947$. As the band structures of Fig 3 (b) show, the new phase $2 \mathrm{H}_{a}-\mathrm{MoS}_{2}$, also semiconducting at low pressures, undergoes a pressure-induced band overlap metallization now near $20 \mathrm{GPa}$. Structurally, $2 \mathrm{H}_{a}$ is less anisotropic than the initial $2 \mathrm{H}_{c}$ structure, the new interlayer spacing (reflected in the c axis length) smaller, the in-plane interatomic spacing (reflected by $a, b$ lengths) larger.

At this point comes an important contact with experiment. Few years ago Aksoy et al. [10] had reported an unspecified structural transformation taking place in $2 \mathrm{H}_{c}-\mathrm{MoS}_{2}$ between 20 and $30 \mathrm{GPa}$, without change of space group symmetry. When we overlap their measured lattice parameters $a$ and $c$ with our calculated ones in Fig,2 the conclusion that their observed transformation is precisely the $2 \mathrm{H}_{c}$ to $2 \mathrm{H}_{a}$ transition strongly suggests itself. To further nail down that conclusion we calculated X-ray powder patterns for $2 \mathrm{H}_{c}-\mathrm{MoS}_{2}$ and $2 \mathrm{H}_{a}-\mathrm{MoS}_{2}$ and compared them in Fig.5 with the measured ones. Again, the agreement is quite good. The pressure induced onset of the $2 \mathrm{H}_{a}$ phase is heralded by the birth of a (104) reflection (indexed as "006" in Ref. [10]) and a much stronger (102) reflection. The simultaneous demise of the $2 \mathrm{H}_{c}-\mathrm{MoS}_{2}$ phase is signaled by the disappearance of (105) and the drop of (103) reflections.

Raman spectroscopy data [11] are also available for $\mathrm{MoS}_{2}$ in the pressure interval up to 

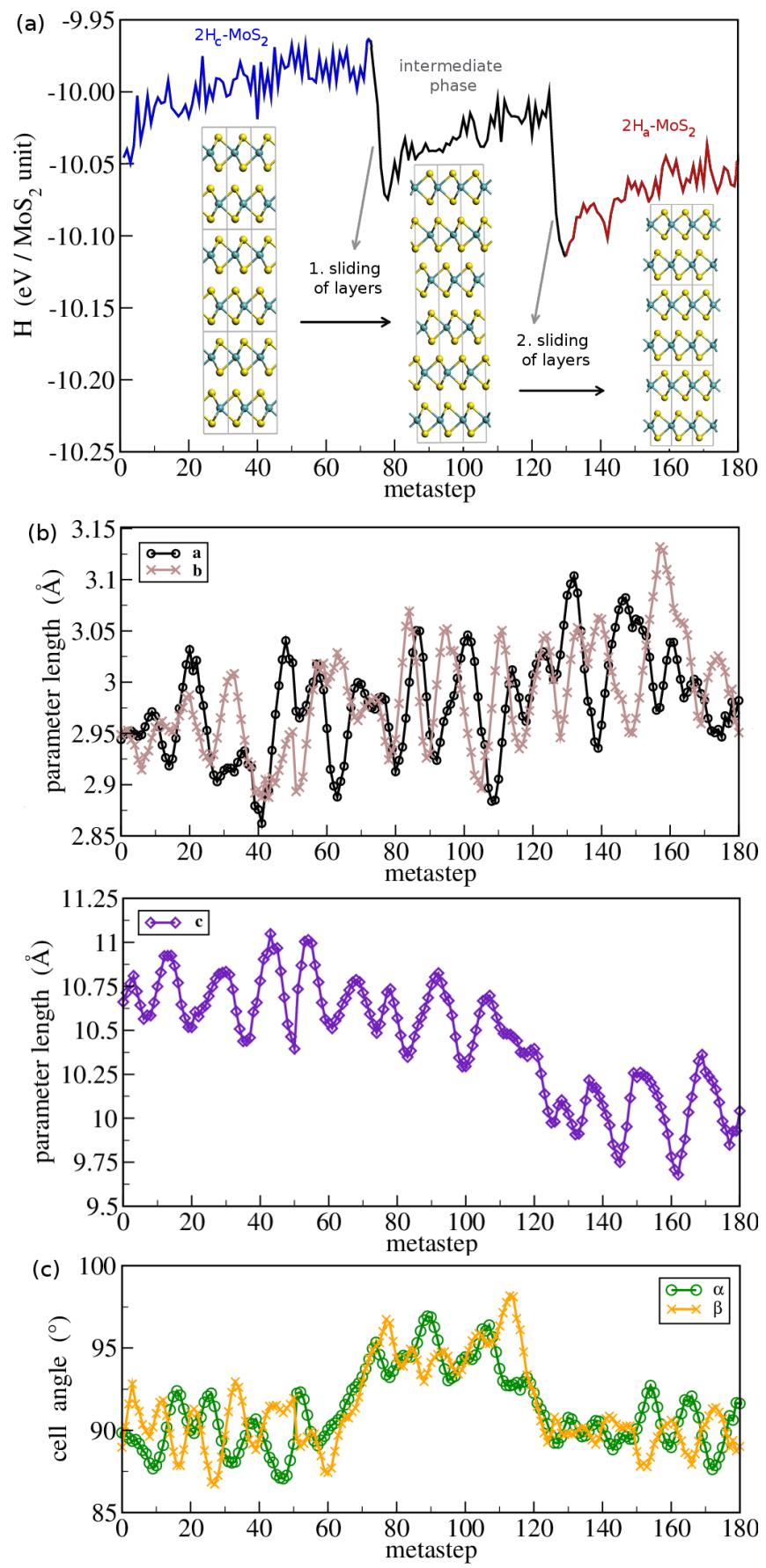

FIG. 4: (a) Evolution of enthalpy in the metadynamics simulation during transformation from $2 \mathrm{H}_{c}-\mathrm{MoS}_{2}$ to $2 \mathrm{H}_{a}-\mathrm{MoS}_{2}$ via an intermediate structure. (b) Evolution of unit cell parameters $a, b, c$. The parameters $a, b$ are equal to the size of the 72-atoms supercell in respective directions divided by $2 \sqrt{3}$. (c) Evolution of supercell angles $\alpha, \beta$. The angle $\gamma$ (not shown) fluctuates around $120^{\circ}$ and does not undergo a significant change. 

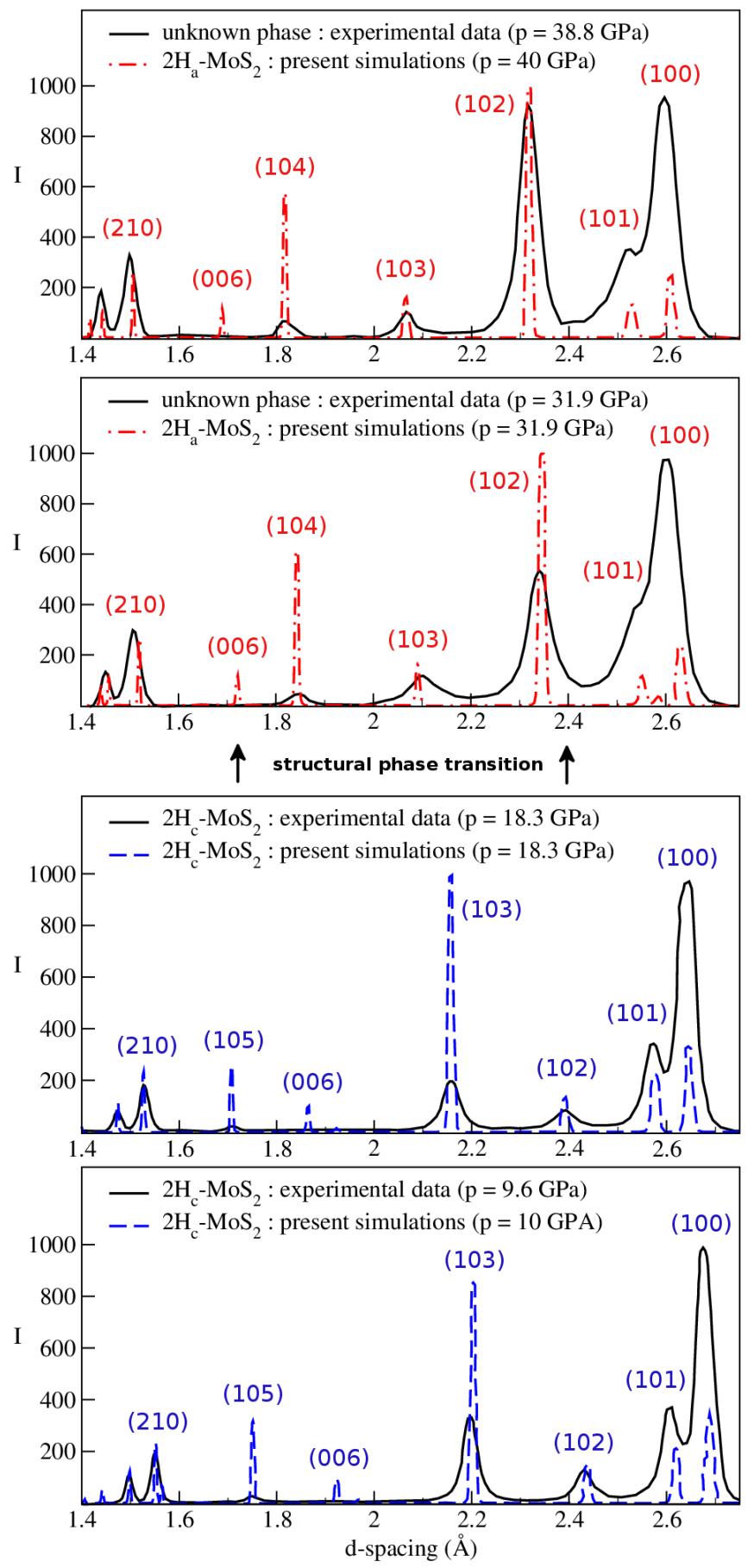

FIG. 5: Comparison of experimental [10] and calculated X-ray diffraction patterns for $2 \mathrm{H}_{c}$ structure below $20 \mathrm{GPa}$ and $2 \mathrm{H}_{a}$ structure above $20 \mathrm{GPa}$.

31 GPa. At $p=19.1$ GPa a new peak (called "d" band, Figs. 5, 6 in Ref.[11]) appears, first as a shoulder on the high-frequency side of the original $\mathrm{E}_{2 g}$ peak. As pressure grows its weight increases at the expense of the original $\mathrm{E}_{2 g}$ peak and at 23.4 and $31 \mathrm{GPa}$ both peaks 
are clearly visible in the spectra. Using the Quantum Espresso code[29] we calculated the frequencies of Raman active $\mathrm{E}_{2 g}$ and $\mathrm{A}_{1 g}$ phonon modes of $2 \mathrm{H}_{c}-\mathrm{MoS}_{2}$ and $2 \mathrm{H}_{a}-\mathrm{MoS}_{2}$ at the same experimental pressures of 19.1, 23.4 and 31 GPa. [34] Comparison with experimental data[11] in Fig, 6 suggests that the "d" band coincides with the $\mathrm{E}_{2 g}$ mode of the emergent $2 \mathrm{H}_{a}$ phase. On the other hand the difference of frequency of the higher $\mathrm{A}_{1 g}$ mode in both phases is very small which explains why this peak is not observed to split in the experiment. Apart from a small systematic difference of about $15 \mathrm{~cm}^{-1}$ between experimental and calculated peak positions the overall agreement is very good. This provides further strong support for the $2 \mathrm{H}_{c} \rightarrow 2 \mathrm{H}_{a}$ structural transition starting at $20 \mathrm{GPa}$ with both phases coexisting up to the highest pressure of $31 \mathrm{GPa}$.

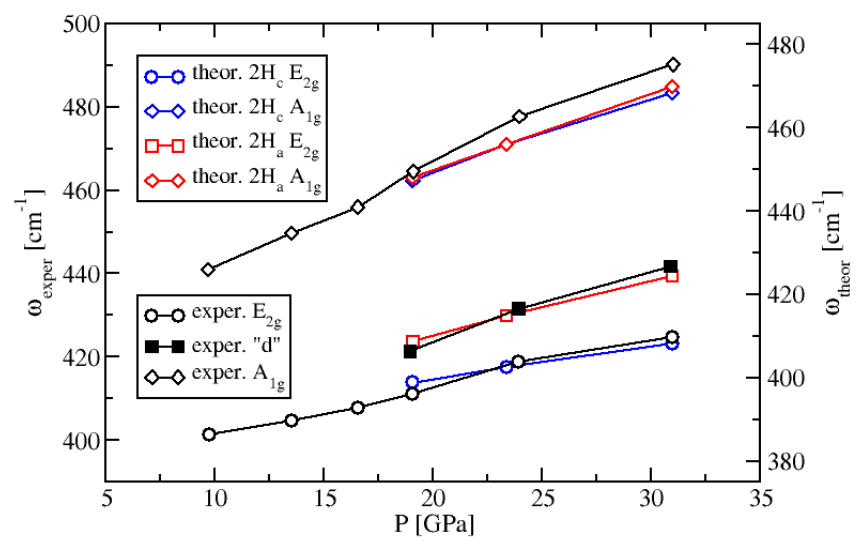

FIG. 6: Comparison of experimental[11] and calculated peak positions of Raman spectra for $2 \mathrm{H}_{c}$ and $2 \mathrm{H}_{a}$ structures at different pressures. The vertical axis for theoretical data (right) has been shifted with respect to the axis for experimental data (left) by $15 \mathrm{~cm}^{-1}$ upwards to account for a small constant difference between the two datasets.

We can now stretch beyond experimental observations, which stop just below 40 GPa, by extending simulations to higher pressures. 35] At $120 \mathrm{GPa}$, searching for newer potential structural transitions, we carried out another metadynamics simulation but the $2 \mathrm{H}_{a}$ structure within the 72-atom supercell remained stable and did not show any transition. We further performed a structural relaxation of the $2 \mathrm{H}_{a}$ structure within the 6-atom unit cell at ultrahigh pressure of $200 \mathrm{GPa}$ and again failed to find any new phase, indicating good local stability of $2 \mathrm{H}_{a}$. 
Having discovered that $\mathrm{MoS}_{2}$ turns metallic above 20-30 GPa, it is a relevant question whether it will superconduct. Inspection of the electronic density of states (DOS) at the Fermi level $n\left(E_{F}\right)$ (Fig. 7) shows that the metallicity of $2 \mathrm{H}_{a}-\mathrm{MoS}_{2}$, marginal near $20 \mathrm{GPa}$, increases only very mildly with pressure. Poor metallicity is probably the reason why Raman spectra are still well defined even at $31 \mathrm{GPa}$. Using again the Quantum Espresso code[29] we calculated besides the DOS, also the dimensionless electron-phonon coupling strength $\lambda$ and the effective logarithmic average phonon frequency $\omega_{l o g}$ at several pressures. As Table II shows, superconducting temperatures estimated by the Allen-Dynes formula [30] are not encouraging. Even for optimistic values for the Coulomb pseudopotential $\mu *$, superconductivity is predicted to appear only at ultrahigh pressures well beyond $100 \mathrm{GPa}$.

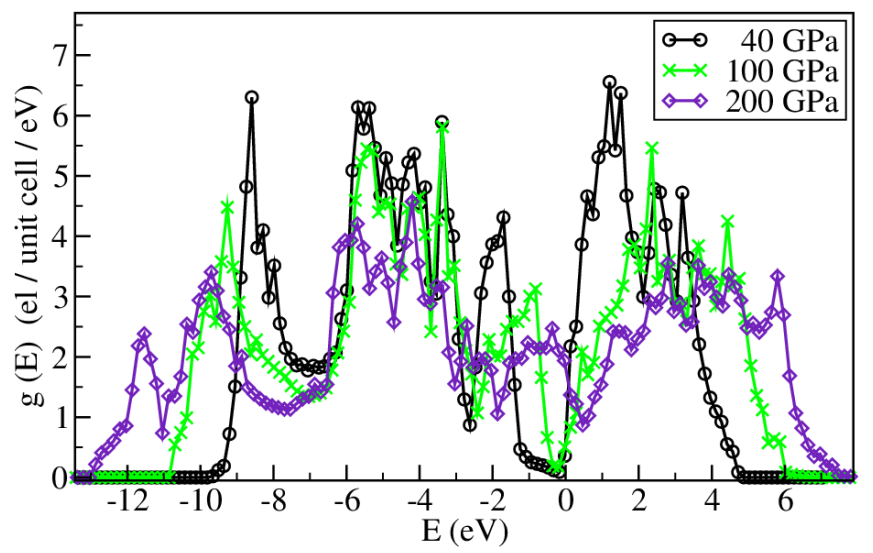

FIG. 7: $2 \mathrm{H}_{a}-\mathrm{MoS}_{2}$ electronic density of states at selected pressures.

TABLE I: Values of $p[\mathrm{GPa}], \lambda, \omega_{l o g}[\mathrm{~K}], n\left(E_{F}\right)$ [electrons/unit cell/eV], $T_{c}\left(\mu^{*}=0.1\right)[\mathrm{K}], T_{c}\left(\mu^{*}=\right.$ $0.05)[\mathrm{K}]$ for $2 \mathrm{H}_{c}-\mathrm{MoS}_{2}$ and $2 \mathrm{H}_{a}-\mathrm{MoS}_{2}$.

\begin{tabular}{|l|l|l|l|l|l|l|}
\hline \hline phase & $p$ & $\lambda$ & $\omega_{l o g}$ & $n\left(E_{F}\right)$ & $T_{c}(0.1)$ & $T_{c}(0.05)$ \\
\hline $2 \mathrm{H}_{c}$ & 40 & 0.095 & 384 & 0.45 & 0.0 & 0.0 \\
$2 \mathrm{H}_{c}$ & 100 & 0.118 & 518 & 0.68 & 0.0 & 0.0 \\
$2 \mathrm{H}_{a}$ & 40 & 0.078 & 388 & 0.44 & 0.0 & 0.0 \\
$2 \mathrm{H}_{a}$ & 100 & 0.108 & 402 & 0.49 & 0.0 & 0.0 \\
$2 \mathrm{H}_{a}$ & 200 & 0.330 & 532 & 1.69 & 0.6 & 2.7 \\
\hline
\end{tabular}

Our overall physical conclusions are therefore that pressure will cause $\mathrm{MoS}_{2}$ layers to slide, causing a structural transition between $2 H_{c}$ and $2 H_{a}$ polytypes, via a process akin 
to superlubric sliding. [31] In both structures, band-overlap metallization will take place at relatively low pressures. After that, however, the broad cleft between valence and conduction bands, both with large Mo $d$-band character, does not shrink fast enough to produce a comparably large metallicity to that generated by, e.g., alkali doping, or by EDL field doping [6, 7]. High pressure $\mathrm{MoS}_{2}$ is predicted to remain semimetallic, and only prone to superconductivity well beyond $100 \mathrm{GPa}$. The contrast with the higher superconducting temperatures obtained by alkali and by EDL electron doping stems first of all from the smaller DOS achievable by pressure, but can also be related to a higher coupling of electrons relative to holes, and to a dimensionality effect in the EDL case. One aspect that remains to be explored is the possible occurrence, to be pursued with methods beyond those currently available, of excitonic insulator driven charge or spin density waves in a narrow pressure range close to the metallization pressure. If such a phase did exist, it is likely to be preceded and/or followed by a superconducting region.

E.T. thanks T. Kagayama, Y. Iwasa, A. Shukla, and M. Calandra for discussions and exchange of information. Work in Trieste was partly sponsored by EU-Japan Project LEMSUPER, and by Sinergia Contract CRSII2 $2_{1} 36287 / 1$. L.H. and R.M were supported by the Slovak Research and Development Agency under Contract No. APVV-0558-10 and by the project implementation 26220220004 within the Research \& Development Operational Programme funded by the ERDF. Part of calculations were performed in the Computing Centre of the Slovak Academy of Sciences using the National Supercomputing Infrastructure supported from Structural Funds of EU. L.H. acknowledges a predoc fellowship held at SISSA during part of this project.

* Electronic address: martonak@fmph.uniba.sk

[1] L. Gmelin, Gmelin Handbook of Inorganic and Organometallic Chemsitry (Springer-Verlag, Berlin, 1995), vol. B 7,8,9, p. 16.

[2] M. Dallavalle, N. Sndig, and F. Zerbetto, Langmuir 28, 7393 (2012).

[3] K. F. Mak, C. Lee, J. Hone, J. Shan, and T. F. Heinz, Phys. Rev. Lett. 105, 136805 (2010).

[4] B. Radisavljevic, A. Radenovic, J. Brivio, V. Giacometti, and A. Kis, Nat. Nano. 6, 147 (2011). 
[5] R. B. Somoano, V. Hadek, and A. Rembaum, The Journal of Chemical Physics 58, 697 (1973).

[6] K. Taniguchi, A. Matsumoto, H. Shimotani, and H. Takagi, Appl. Phys. Lett. 101, 042603 (2012).

[7] J. T. Ye, Y. J. Zhang, R. Akashi, M. S. Bahramy, R. Arita, and Y. Iwasa, Science 338, 1193 (2012).

[8] S. Bhattacharyya and A. K. Singh, Phys. Rev. B 86, 075454 (2012).

[9] M. Dave, R. Vaidya, S. G. Patel, and A. R. Jani, Bull. Mater. Sci. 27, 213216 (2004).

[10] R. Aksoy, Y. Ma, E. Selvi, M. C. Chyu, A. Ertas, and A. White, Journal of Physics and Chemistry of Solids 67, 1914 (2006).

[11] T. Livneh and E. Sterer, Phys. Rev. B 81, 195209 (2010).

[12] R. Martoňák, A. Laio, and M. Parrinello, Phys. Rev. Lett. 90, 075503 (2003).

[13] R. Martoňák, D. Donadio, A. R. Oganov, and M. Parrinello, Nature Materials 5, 623 (2006).

[14] R. Martoňák, in Modern methods of crystal structure prediction, edited by A. R. Oganov (Wiley-VCH, Berlin, 2011).

[15] R. Martoňák, Eur. Phys. J. B 79, 241252 (2011).

[16] G. Kresse and J. Furthmüller, Phys. Rev. B 54, 11169 (1996).

[17] G. Kresse and D. Joubert, Phys. Rev. B 59, 1758 (1999).

[18] H. J. Monkhorst and J. D. Pack, Phys. Rev. B 13, 5188 (1976).

[19] J. P. Perdew, K. Burke, and M. Ernzerhof, Phys. Rev. Lett. 77, 3865-3868 (1996).

[20] A. D. Becke, J. Chem. Phys. 98, 5648 (1993).

[21] P. J. Stephens, F. J. Devlin, C. F. Chabalowski, and M. J. Frisch, The Journal of Physical Chemistry 98, 11623 (1994).

[22] A. V. Krukau, O. A. Vydrov, A. F. Izmaylov, and G. E. Scuseria, The Journal of Chemical Physics 125, 224106 (pages 5) (2006).

[23] S. Grimme, Journal of Computational Chemistry 27, 1787 (2006), ISSN 1096-987X.

[24] A. Kokalj, Comp. Mater. Sci. 28, 155 (2003), URL http://www.xcrysden.org.

[25] H. Guo, T. Yang, P. Tao, Y. Wang, and Z. Zhang (2012), URL http://arxiv.org/abs/ $1208.5941 \mathrm{v} 2$,

[26] D. Jérome, T. M. Rice, and W. Kohn, Phys. Rev. 158, 462 (1967).

[27] H. Katzke, P. Tolédano, and W. Depmeier, Phys. Rev. B 69, 134111 (2004).

[28] F. E. Wickman and D. K. Smith, The American Mineralogist 55 (1970). 
[29] P. Giannozzi, S. Baroni, N. Bonini, M. Calandra, R. Car, C. Cavazzoni, D. Ceresoli, G. L. Chiarotti, M. Cococcioni, I. Dabo, et al., J.Phys.:Condens.Matter 21, 395502 (2009).

[30] P. B. Allen and R. C. Dynes, Phys. Rev. B 12, 905 (1975).

[31] A. Vanossi, N. Manini, M. Urbakh, S. Zapperi, and E. Tosatti, Rev. Mod. Phys., in press (2012), URL http://arxiv.org/abs/1112.3234.

[32] We adopt here the notation of Toledano et al.[27], while in earlier works such as, e.g., Ref.[28], this structure was denoted as $2 \mathrm{H}_{b}$.

[33] Zero-point motion and entropic contributions for both phases were estimated within the quasiharmonic approximation at $p=10 \mathrm{GPa}$ and $T=300 \mathrm{~K}$. The difference between the two phases was smaller than $1 \mathrm{meV} /$ atom and therefore these contributions were neglected in the figure.

[34] We used the PBE PAW pseudopotentials Mo.pbe-spn-kjpaw.UPF and S.pbe-n-kjpaw.UPF.

[35] Above 50 GPa we used the hard version of VASP PAW pseudopotentials. 